\title{
Statistical and theoretical studies of flares from Sagittarius $\mathbf{A}^{\star}$
}

\author{
Ya-Ping $\mathbf{L i}^{1,2}$, Qiang Yuan ${ }^{3,4}$, Q. Daniel Wang ${ }^{4}$, P. F. Chen ${ }^{5}$, \\ Joseph Neilsen ${ }^{6}$, Taotao Fang ${ }^{2}$, Shuo Zhang ${ }^{7}$ and Jason Dexter ${ }^{8}$ \\ ${ }^{1}$ Shanghai Astronomical Observatory, Chinese Academy of Sciences, 80 Nandan Road, \\ Shanghai 200030, China \\ email: leeyp2009@gmail.com \\ ${ }^{2}$ Department of Astronomy and Institute of Theoretical Physics and Astrophysics, Xiamen \\ University, Xiamen, Fujian 361005, China \\ ${ }^{3}$ Key Laboratory of Dark Matter and Space Astronomy, Purple Mountain Observatory, \\ Chinese Academy of Science, Nanjing, 210008, China \\ ${ }^{4}$ Department of Astronomy, University of Massachusetts, Amherst, MA 01003, USA \\ ${ }^{5}$ School of Astronomy and Space Science, Nanjing University, Nanjing 210023, China \\ ${ }^{6}$ MIT Kavli Institute for Astrophysics and Space Research, Cambridge, MA 02139, USA \\ ${ }^{7}$ Columbia Astrophysics Laboratory, Columbia University, New York, NY 10027, USA \\ ${ }^{8}$ Max Planck Institute for Extraterrestrial Physics, P.O. Box 1312, Giessenbachstr., D-85741 \\ Garching, Germany
}

\begin{abstract}
Multi-wavelength flares have routinely been observed from the supermassive black hole, Sagittarius $A^{\star}\left(\mathrm{Sgr} \mathrm{A}^{\star}\right)$, at our Galactic center. The nature of these flares remains largely unclear, despite many theoretical models. We study the statistical properties of the Sgr $\mathrm{A}^{\star} \mathrm{X}$ ray flares and find that they are consistent with the theoretical prediction of the self-organized criticality system with the spatial dimension $S=3$. We suggest that the X-ray flares represent plasmoid ejections driven by magnetic reconnection (similar to solar flares) in the accretion flow onto the black hole. Motivated by the statistical results, we further develop a time-dependent magnetohydrodynamic (MHD) model for the multi-band flares from Sgr $\mathrm{A}^{\star}$ by analogy with models of solar flares/coronal mass ejections (CMEs). We calculate the X-ray, infrared flare light curves, and the spectra, and find that our model can explain the main features of the flares.
\end{abstract}

Keywords. Galaxy: center — black hole physics - accretion, accretion disks - methods: statistical - MHD

\section{Introduction}

Sagittarius $A^{\star}\left(\operatorname{Sgr} A^{\star}\right)$ at the center of the Milky Way is an excellent laboratory for studying the accretion and ejection of matter by supermassive black holes (SMBHs). There have been quite a number of observational and theoretical studies of Sgr $\mathrm{A}^{\star}$ (see reviews by Genzel et al. 2010 and Yuan \& Narayan 2014). The bolometric luminosity of Sgr $A^{\star}$ is $L_{\mathrm{bol}} \sim 10^{-9} L_{\mathrm{Edd}}$ (where $L_{\mathrm{Edd}}$ is the Eddington luminosity), which is five orders of magnitude lower than that predicted by a standard thin disk accretion at the Bondi accretion rate (Baganoff et al. 2003). We now understand that an advection-dominated accretion flow scenario works for $\operatorname{Sgr} A^{\star}$, and that the low luminosity is due to the combination of the low radiative efficiency and the mass loss via outflow (see review by Yuan \& Narayan 2014, and references therein).

Sgr $\mathrm{A}^{\star}$ is usually in a quiescent state, and occasionally shows rapid flares (on time scales $\sim 1$ hour), most significantly in X-ray (Baganoff et al. 2001) and near-infrared (NIR; 
Genzel et al. 2003, Ghez et al. 2004), but less prominent in radio and sub-millimeter. As we review in $\S 2$, numerous observational and theoretical efforts have been made since the first detection of flares from $\mathrm{Sgr} \mathrm{A}^{\star}$. However, first-principle understanding of the flaring nature from Sgr $\mathrm{A}^{\star}$ is still lacking since most of the theoretical works are phenomenological.

In this work, with the aim to understand the multi-wavelength flaring nature of Sgr $\mathrm{A}^{\star}$ we first present a statistical analysis of X-ray flare data to reveal its energetic nature ( $\mathrm{Li}$ et al. 2015). Motivated by the statistical results, we further propose a magnetohydrodynamic (MHD) model, by analogy with solar flares/coronal mass ejections (CMEs), to interpret the temporal and spectral properties of multi-wavelength flares ( $\mathrm{Li}$ et al. 2016, in preparation).

\section{Observational and Theoretical Progress for Flares from $\mathrm{Sgr} \mathbf{A}^{\star}$}

Since the first flare detections of $\operatorname{Sgr} \mathrm{A}^{\star}$ in the X-ray and NIR, a number of multiwavelength campaigns have been conducted routinely to monitor the flares from Sgr $\mathrm{A}^{\star}$. We briefly summary the main properties of the multi-wavelength flares (see also DoddsEden et al. (2009) for a detailed summary of the general properties of the NIR and X-ray flares from Sgr $\mathrm{A}^{\star}$ ). The flare rate is roughly once per day in X-ray (Neilsen et al. 2013; Ponti et al. 2015; Yuan \& Wang 2016) and more frequently in NIR. The NIR and X-ray flares occur simultaneously within 3 mins when both are observed in company (but see Yusef-Zadeh et al. (2012) for a counter-example). The luminosity excursion can be up to a factor of $\sim 20$ above the detection limit in the NIR and up to a factor of $\sim 400$ in X-ray (Haggard et al., this proceedings volume). The full width at half maximum (FWHM) of the NIR flare profile is about 60 mins, which is about twice as broad as that of the X-ray. There are substructure variations with characteristic timescale of $\sim 20$ mins in the NIR light curve occasionally, but not present at the same level in X-ray. Both X-ray and NIR flares are relatively symmetric, but the brightest flare in Nowak et al. (2012) shows a remarkable asymmetry profile with a faster decline than rise. A large fraction of the X-ray flares in the Chandra X-ray Visionary Project on Sgr A* (hereafter XVP) campaign also shows a faster rise and slow decay profile (Yuan et al. 2016). The flares in the NIR are significantly polarized with the typical degrees of polarization of the order of $20 \pm 10 \%$ (e.g., Shahzamanian et al. 2015). Concerning flares at the longer wavelength, the light curves in sub-millimeter and radio show much shallower and broader compared to NIR and X-ray. There is a general trend that the peak flare emission at higher radio frequency leads that of a lower one, e.g., $43 \mathrm{GHz}$ leading $22 \mathrm{GHz}$ by $20 \sim 40$ mins (Yusef-Zadeh et al. 2006b). Time lags are also detected among radio, sub-millimeter and NIR/X-ray flares (e.g., Marrone et al. 2008; Brinkerink et al. 2015).

Irrespective of addressing the energetic nature driving the flares, many efforts have been made to understand the underlying emission mechanism. The proposed flare models usually invoke synchrotron (e.g., Yuan et al. 2003; Dodds-Eden et al. 2009) or inverse Compton (e.g., Yuan et al. 2003; Yusef-Zadeh et al. 2012) radiation processes. The highly polarized NIR emission is the evidence of a synchrotron origin of the NIR flares, produced by a population of non-thermal electrons (e.g., Eckart et al. (2006a), Shahzamanian et al. (2015), see also references therein). The non-thermal electrons are likely accelerated by magnetic reconnection, shock or turbulence in either an accretion flow (e.g., Li et al. 2015, and references therein) or an assume jet (Markoff et al. 2001).

Theoretical interpretations of the flares involving the energetic nature include accretion instability (Tagger \& Melia 2006; Falanga et al. 2008), orbiting hot spots (e.g., Trippe et al. 2007; Hamaus et al. 2009), expanding plasma blobs (e.g., Yusef-Zadeh et al. 2006b; 
Dodds-Eden et al. 2010), and tidal disruption of asteroids by the SMBH (e.g., Čadež et al. 2008; Zubovas et al. 2008). There are only several works investigating the time-dependent emission in detail instead focusing on the average properties of the flares (Dexter et al. 2009; Maitra et al. 2009; Dodds-Eden et al. 2010; Kusunose \& Takahara 2011; Chan et al. 2015). The nature of these flares remains largely unclear, despite many theoretical works.

\section{Statistical Studies of X-ray Flares from Sgr $\mathbf{A}^{\star}$}

\subsection{Data and Methodology}

Observations: We use the data of the 2012 Chandra XVP campaign, which consists of 38 Chandra ACIS-S/HETGS observations of Sgr $\mathrm{A}^{\star}$ with the total exposure time of 3 million seconds (Ms) between February 6 and October 29 in 2012. For more details of the XVP campaign and the data reduction, we refer the readers to Neilsen et al. (2013).

Methodology: The light curve can be decomposed into the quiescent and flare components. The quiescent emission is assumed to be steady with a count rate (CR) $r$. We model the flare component as a sum of Gaussian functions. There are four parameters to quantify the flare distribution, the power-law index of the fluence distribution $\alpha_{\mathrm{E}}$, the total flare number $\kappa$, the duration normalization $A$, and the fluence-duration correlation slope $\alpha_{\mathrm{ET}}$. We use a Monte Carlo method to generate the synthetic X-ray light curve, which accounts for the Possion fluctuations of photon counting. In total we will have five parameters to describe the synthetic light curve, namely $r, \alpha_{\mathrm{E}}, \kappa, A$, and $\alpha_{\mathrm{ET}}$.

The synthetic light curve cannot be directly compared to the observational one due to the lack of bin-to-bin correspondence between the two. We need a statistical way to make the comparison. We use the CR distribution as a "first" order statistics, and the structure function (SF) to account for the flare flux correlation among adjacent bins. We jointly fit the light curve to constrain the five model parameters by minimizing $\chi^{2}=\chi_{\mathrm{CR}}^{2}+\chi_{\mathrm{SF}}^{2}$ (where $\chi_{\mathrm{CR}}^{2}$ and $\chi_{\mathrm{SF}}^{2}$ are the $\chi^{2}$ statistics for the CR distribution and the SF), using the MCMC method (see Li et al. 2015 for details).

\subsection{Results}

Figure 1 shows the one and two dimensional (1D and 2D) probability distributions of the parameters. The fitting results suggest a fluence distribution power-law index $\alpha_{\mathrm{E}}=$ $1.65 \pm 0.17$, and the less constrained fluence-duration correlation index $\alpha_{\mathrm{ET}}<0.55 \quad(95 \%$ confidence limit). The correlations between some of the parameters can be clearly seen in Figure 1. There is a strong correlation between the total number of flares $\kappa$ and the fluence distribution index $\alpha_{\mathrm{E}}$. It is easy to understand that a harder fluence distribution will naturally correspond to a smaller number of flares in order not to over-produce the number of photons. Also the anti-correlation between $\alpha_{\mathrm{E}}$ (or $\kappa$ ) and $r$ is again due to the constraint of the total number of photon counts.

\subsection{Implications on the Nature of the Flares}

We find that both the flare fluence distribution and the correlation between the duration and the fluence can be described by power-laws, which is consistent with the prediction of the fractal-diffusive self-organized criticality (SOC) theory (Aschwanden 2011). SOC describes a class of dynamical system with nonlinear energy dissipation that is slowly and continuously driven toward a critical value of an instability threshold (Katz 1986; Bak et al. 1987). The SOC theory further predicts that the power-law indices will depend on the Euclidean space dimension $S$ of the system to produce flares. The predicted powerlaw indices are $\alpha_{\mathrm{E}}^{\text {th }} \approx 1.5$ and $\alpha_{\mathrm{ET}}^{\text {th }} \approx 0.5$, respectively, for $S=3$, the classical diffusion 

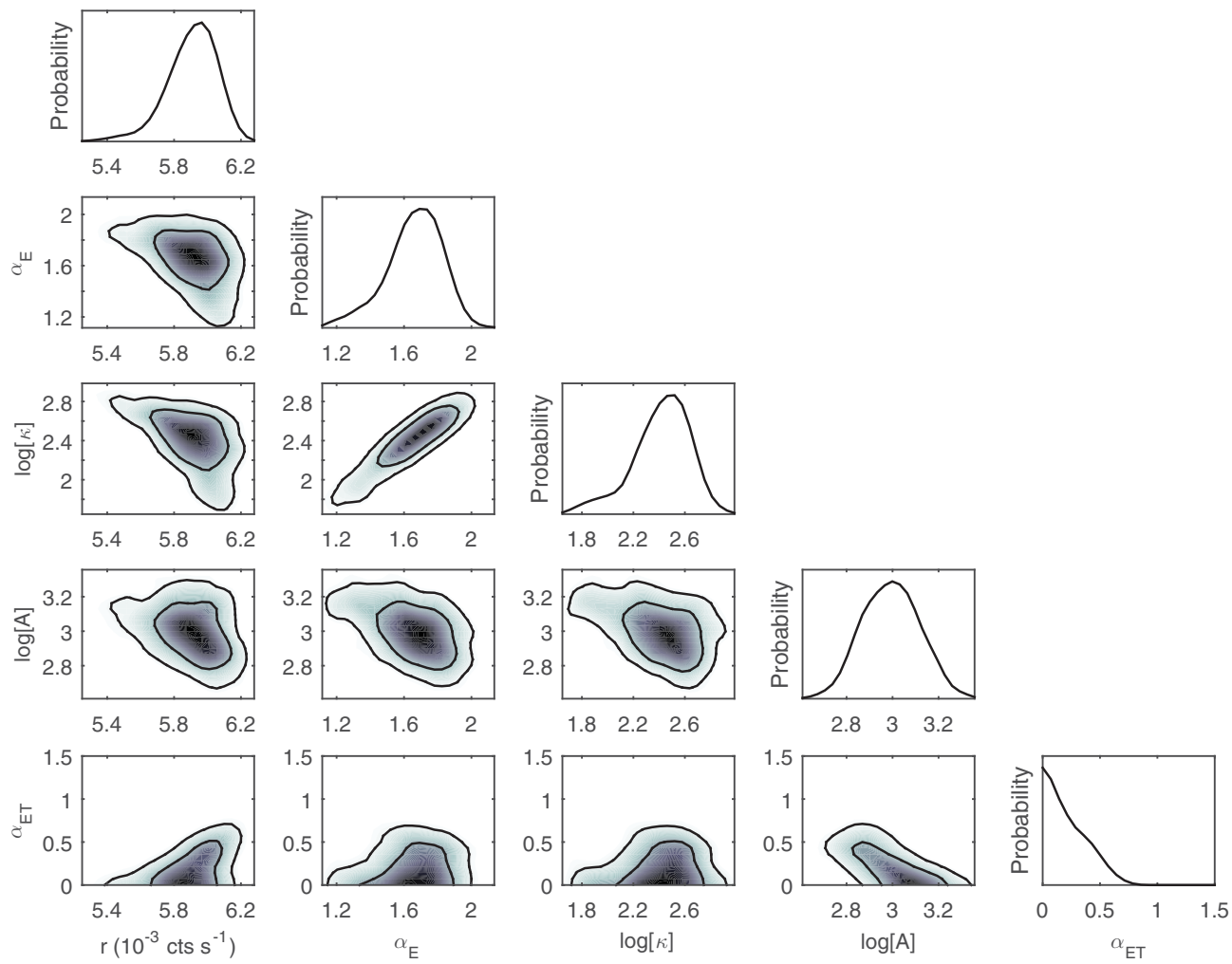

Figure 1. 1D and 2D probability distributions of the fitting parameters. The contours in the 2D plots are for confidence levels $68 \%$ and $95 \%$ from inside to outside, respectively.

parameter $\beta=1$ and the mean fractal dimension $D_{\mathrm{S}}=(1+S) / 2$. Our results of $\alpha_{\mathrm{E}}$ and $\alpha_{\mathrm{ET}}$ are actually in good agreement with the theoretical expectation with $S=3$. The predicted indices of the duration and peak rate distributions are $\alpha_{\mathrm{T}}^{\mathrm{th}} \approx 2.0$ and $\alpha_{\mathrm{P}}^{\mathrm{th}} \approx 1.7$. As a comparison, our induced values are $\alpha_{\mathrm{T}} \gtrsim 2.1$ and $1.7 \lesssim \alpha_{\mathrm{P}} \lesssim 2.4$, respectively. There are potential discrepancies of these two distributions. Since the determination of the peak rate depends on the assumption of the flare profile, as well as the precise measurement of the flare duration, there should be relatively large uncertainty of the peak CR distribution. As shown in Nowak et al. (2012), the profile, at least for the bright ones, is indeed asymmetric rather than Gaussian. Therefore, the integral property (fluence) should be more reliably measured and more suitable to be used to compare with the theoretical model expectation.

Two main conclusions can thus be obtained from the above analysis. First, the powerlaw distributions of the fluences, durations, and their correlation, suggest that the flares of Sgr $\mathrm{A}^{\star}$ can be explained in the fractal-diffusive SOC framework. Second, the inferred space dimension responsible for the flares is $S=3$. Both results are similar to the solar flares, which thus implies that the X-ray flares of Sgr $A^{\star}$ are likely driven by a mechanism similar to that of the solar flares, i.e., magnetic reconnection. The three-dimensional geometry of the energy dissipation domain further suggests that the X-ray flaring of Sgr $A^{\star}$ occurs in the surface of the accretion flow due to the less-ordered magnetic field structure embedded in it compared with that in the relativistic astrophysical jets (Wang \& Dai 2013). There are also two pieces of supporting evidence for our conclusion. On the one hand, the flares and episodic ejections are physically associated with each other, 
both for the Sun and Sgr $A^{\star} \dagger$. On the other hand, the recent development of magnetohydrodynamic simulations leads to the consensus that the accretion flow in Sgr $A^{\star}$ is enveloped by a tenuous corona above the dense disk (see Yuan \& Narayan 2014 for a review), similar to the atmosphere of the Sun.

\section{Theoretical Modeling of Multi-wavelength Flares from Sgr $\mathbf{A}^{\star}$}

Motivated by the statistical results of X-ray flares from $\mathrm{Sgr} \mathrm{A}^{\star}$, we propose an MHD model for the flare production. By analogy of CMEs and their solar flares, Yuan et al. (2009) have proposed a magnetic reconnection model for the episodic ejections from the accretion flow of Sgr $A^{\star}$. Here we mainly focus on the radiative manifestation of the catastrophic system instead of the dynamical nature of the plasmoid ejection as in Yuan et al. (2009). As the radiative flares are associated with the dynamical evolutions of the system, we first elaborate how the system evolves in the framework of the CME (Lin \& Forbes 2000; Yuan et al. 2009; Chen 2011), which is mainly described by the dynamical process of plasmoid (flux rope) from the accretion disk/corona (see Li et al. 2016 for details).

The motion of the flux rope is governed by magnetic and gravitational forces, which are described as

$$
m \gamma_{\mathrm{b}}^{3} \frac{d^{2} h}{d t^{2}}=\frac{1}{c}\left|\boldsymbol{I} \times \boldsymbol{B}_{\text {ext }}\right|-F_{\mathrm{g}}
$$

to the first order of approximation, where $m$ is the total mass inside the flux rope per unit length, $\gamma_{\mathrm{b}}=1 / \sqrt{1-\dot{h}^{2} / c^{2}}$ is the Lorentz factor of the radiative blob, $h$ is the height of the flux rope from the surface of the accretion flow, $\boldsymbol{I}$ is the total electric current intensity flowing inside the flux rope, $\boldsymbol{B}_{\text {ext }}$ is the total external magnetic field measured at the center of the flux rope, and $F_{\mathrm{g}}$ is the gravitational force acting on the mass inside the flux rope. Solving this equation will give the dynamical properties of the system following the catastrophe. We show the dynamical properties of the system in the right panel of Figure 2. It is clear that the flux rope can be accelerated to mid-relativistic speed within several minutes and the height of the flux rope can be as high as several hundred $r_{\mathrm{g}}$. Such a catastrophic behaviour is consistent with our SOC predication in $\S 3.3$.

The evolution profile of the force-free magnetic field in the system then can be obtained once we know the dynamic properties. The role of the magnetic field is twofold. First, it determines the energy dissipation rate in the current sheet region due to magnetic reconnection. Second, as two cooling processes, synchrotron and adiabatic cooling, are needed to be considered in the radiative calculation, the strength of the magnetic field can have a large impact on the synchrotron cooling rate. The adiabatic cooling rate can be self-consistently taken into account by the dynamical evolution of the two flare regions indicated in the left panel of Figure 2.

Based on Poynting's theorem, the change rate of the thermal energy, which is a part of the magnetic energy, is determined by the integral of the Poynting flux along the current sheet $S(t)$ (Reeves \& Forbes 2005). It is this part of magnetic energy that contributes to the observed radiation in the eruption. By calculating the Poynting flux in the current sheet, we can thus obtain the power related to the energy dissipated in the current sheet. Given $p, q, h$, and $\dot{h}$ as a function of time, we then obtain the power associated

$\dagger$ The time delay between different radio flare is the evidence of the plasmoid blob ejection from Sgr A* (e.g., Yusef-Zadeh et al. 2006b). 

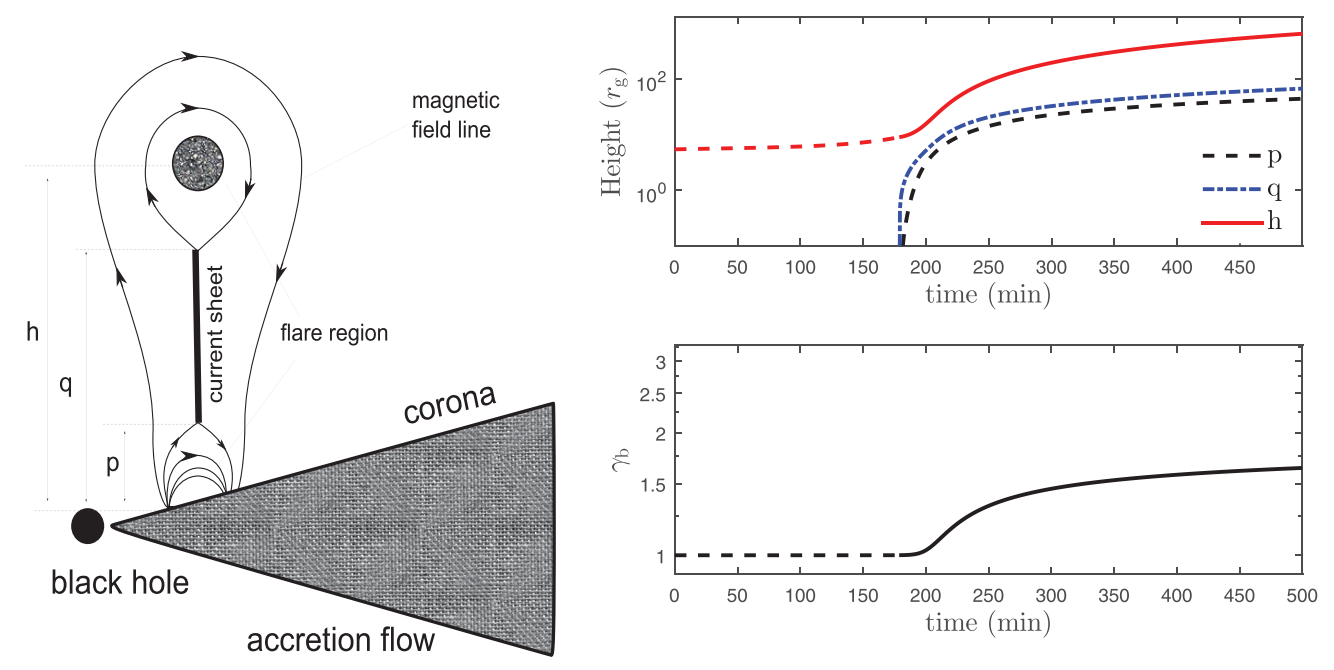

Figure 2. Left panel: Diagram of the flux rope configuration, showing the mathematical notation used in the text. The $x$-axis is parallel to the equatorial plane of the accretion flow, and the $y$-axis points upward. The bottom, top tip of the current sheet, and the height of the center of the flux rope are denoted by $p, q$, and $h$, respectively. All these heights are measured from the disk surface in our calculations. The distance between the magnetic source regions on the accretion flow is $2 \lambda$. Right panel (Upper): the red solid, blue, and black lines present the evolutions of the height of the flux rope $h$, and the current sheet $(q, p)$ as a function of time. The red dashed line corresponds to the evolution prior to the loss of the equilibrium. Right panel (Bottom): the evolution of the Lorentz factor $\gamma_{\mathrm{b}}$ of the flux rope.

with the dynamical evolution, most of which will heat the thermal electrons flowing into two flare regions. We assume that the injected energy in non-thermal (i.e., power-law) electrons is equal to a fraction $\eta$ of the energy in thermal electrons. The normalization $c_{\text {inj }}$ and the minimum Lorentz factor $\gamma_{\text {min }}$ of power-law electrons then can be obtained with the assumption that the injected power-law index $p_{\mathrm{e}}$ being constant. Solving the continuity equation for electrons, we have a time-dependent electron energy distribution after considering the two cooling processes. The final synchrotron light curves for NIR and X-ray flares are shown in Figure 3, and the spectral energy distribution (SED) with the same model parameters is presented in Figure 4. The main characteristic of NIR and X-ray flares, i.e., the luminosity, duration, simultaneousness, can be well produced within our MHD models. Some other features, for example, the substructures, asymmetry, polarizations, and time delay of flares between different radio bands mentioned in the $\S 2$, will be investigated in a separate work.

\section{Summary}

In this paper, we first briefly review current observational and theoretical understanding for multi-band flares from Sgr A*. Although many theoretical efforts have been made, the nature of flares is still largely uncertain.

We then present a statistical analysis to the Chandra $2-8 \mathrm{keV}$ X-ray light curve of Sgr $\mathrm{A}^{\star}$ from the $3 \mathrm{Ms}$ XVP campaign. A Monte Carlo simulation method is adopted to generate the model light curves. Then we fit the $\mathrm{CR}$ distribution and the SF of the light curves jointly to constrain the model parameters, via an MCMC method. We find that the X-ray flare emission of Sgr $\mathrm{A}^{\star}$ can be well modelled by a power-law fluence distribution $d N / d E \propto E^{-\alpha_{\mathrm{E}}}$ with $\alpha_{\mathrm{E}}=1.65 \pm 0.17$. The duration-fluence correlation 

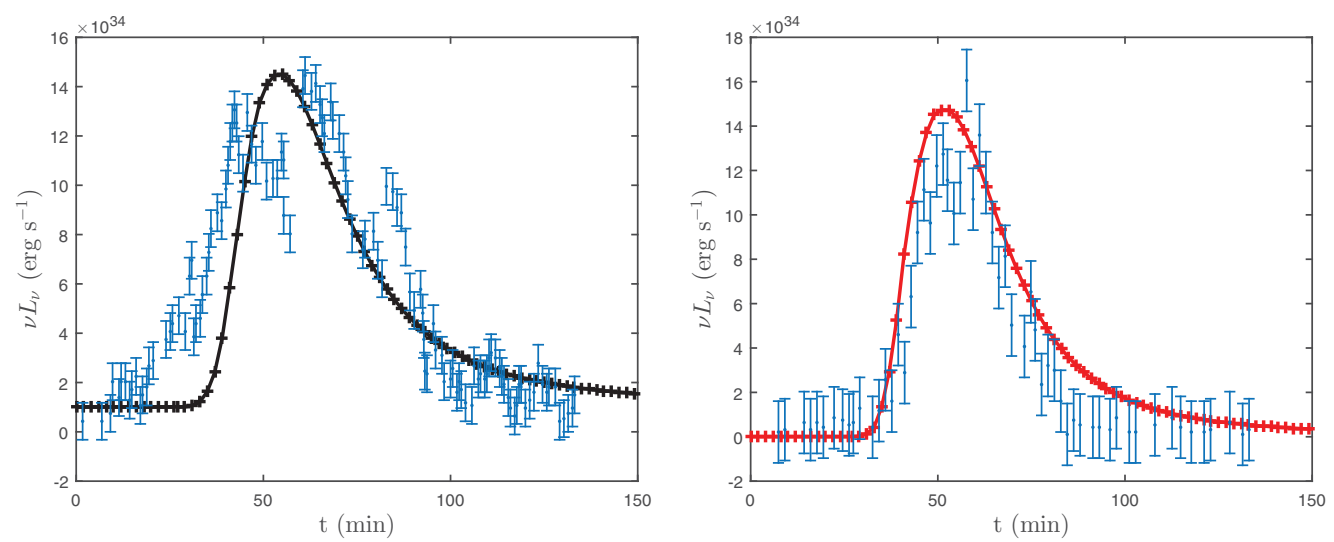

Figure 3. NIR and X-ray flare light curves of $\operatorname{Sgr} A^{\star}$. Left panel: the black solid line is the theoretical NIR light curve. For comparison, the blue points with error bars present the observational data on 2007 April 4 (Dodds-Eden et al. 2009). Right panel: theoretical X-ray light curve (red) and the observed one (blue) during the same period.

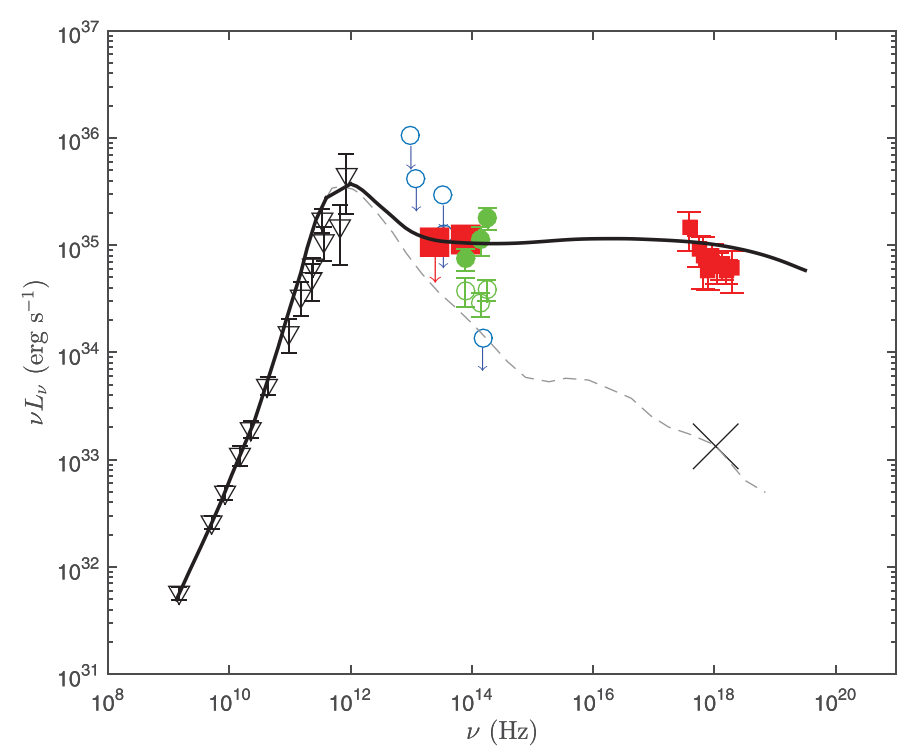

Figure 4. SED of Sgr $A^{\star}$. The observed data are quoted from Dodds-Eden et al. (2009). The filled red and green symbols present the flare state emission in infrared and X-ray, other data points correspond to the quiescent emission. The gray dashed line shows the RIAF model for the quiescent state emission from Yuan et al. (2003). The thick solid black line is the total model SED from synchrotron emission for Sgr $\mathrm{A}^{\star}$ after taking account of the quiescent emission.

can also be modelled by a power-law form $T \propto E^{\alpha_{\mathrm{ET}}}$ with $\alpha_{\mathrm{ET}}<0.55$. These statistical properties are consistent with the theoretical predications of the SOC system with the Euclid spatial dimension $S=3$, the same as that for solar flares (Aschwanden 2012). Our analysis, therefore, indicates that the X-ray flares of Sgr $\mathrm{A}^{\star}$ are possibly driven by the same physical mechanism as that of the solar flares, i.e., magnetic reconnection.

Motivated by the statistical results, we develop a theoretical model via an analytic approach for the flares of $\mathrm{Sgr} \mathrm{A}^{\star}$ in the framework of the solar CME catastrophe model. We first elaborate how the plasmoid (flux rope) evolves and propagates from the accretion disk. The plasmoid is in an equilibrium state initially where there is a balance between 
magnetic and gravity forces. Due to the turbulence and differential rotation nature of the accretion flow, the equilibrium of the plasmoid is broken down and rapidly thrusts outwards. Accompanying this catastrophic process, the magnetic energy accumulated in the previous stage is released to power the radiative flares due to magnetic reconnection. By assuming a constant fraction of magnetic power converted into the power-law electrons, the resultant synchrotron emissions are calculated and compared with the observed flare light curves in NIR and X-ray bands. The theoretical SED can also be obtained to constrain the model parameters. Our numerical results are in good agreement with both the timing and spectral properties of NIR and X-ray flares from Sgr $A^{\star}$.

\section{References}

Aschwanden, M. J. 2012, A\&SA, 539, A2

Aschwanden, M. J. 2011, Self-Organized Criticality in Astrophysics, by Markus J. Aschwanden. Springer-Praxis, Berlin ISBN 978-3-642-15000-5, 416p.

Baganoff, F. K., Bautz, M. W., Brandt, W. N., et al. 2001, Nature, 413, 45

Bak, P., Tang, C., \& Wiesenfeld, K. 1987, Phys. Rev. Lett., 59, 381

Brinkerink, C. D., Falcke, H., Law, C. J., et al. 2015, A\&A, 576, A41

Čadež, A., Calvani, M., \& Kostić, U. 2008, A\& $A, 487,527$

Chan, C.-k., Psaltis, D., Özel, F., et al. 2015, ApJ, 812, 103

Chen, P. F. 2011, Living Reviews in Solar Physics, 8, 1

Dexter, J., Agol, E., \& Fragile, P. C. 2009, ApJL, 703, L142

Dodds-Eden, K., Porquet, D., Trap, G., et al. 2009, ApJ, 698, 676

Dodds-Eden, K., Sharma, P., Quataert, E., et al. 2010, ApJ, 725, 450

Eckart, A., Schödel, R., Meyer, L., et al. 2006a, A\&A, 455, 1

Falanga, M., Melia, F., Prescher, M., Bélanger, G., \& Goldwurm, A. 2008, ApJL, 679, L93

Genzel, R., Schödel, R., Ott, T., et al. 2003, Nature, 425, 934

Genzel, R., Eisenhauer, F., \& Gillessen, S. 2010, Rev. Mod. Phys., 82, 3121

Ghez, A. M., Wright, S. A., Matthews, K., et al. 2004, ApJL, 601, L159

Hamaus, N., Paumard, T., Müller, T., et al. 2009, ApJ, 692, 902

Katz, J. I. 1986, JGR, 91, 10412

Kusunose, M. \& Takahara, F. 2011, ApJ, 726, 54

Li, Y.-P., Yuan, F., Yuan, Q., et al. 2015, ApJ, 810, 19

Lin, J. \& Forbes, T. G. 2000, JGR, 105, 2375

Maitra, D., Markoff, S., \& Falcke, H. 2009, A\&A, 508, L13

Markoff, S., Falcke, H., Yuan, F., \& Biermann, P. L. 2001, A\& A, 379, L13

Marrone, D. P., Baganoff, F. K., Morris, M. R., et al. 2008, ApJ, 682, 373

Neilsen, J., Nowak, M. A., Gammie, C., et al. 2013, ApJ, 774, 42

Nowak, M. A., Neilsen, J., Markoff, S. B., et al. 2012, ApJ, 759, 95

Ponti, G., De Marco, B., Morris, M. R., et al. 2015, MNRAS, 454, 1525

Reeves, K. K. \& Forbes, T. G. 2005, ApJ, 630, 1133

Shahzamanian, B., Eckart, A., Valencia-S., M., et al. 2015, A\&SA, 576, A20

Tagger, M. \& Melia, F. 2006, ApJL, 636, L33

Trippe, S., Paumard, T., Ott, T., et al. 2007, MNRAS, 375, 764

Wang, F. Y. \& Dai, Z. G. 2013, Nature Physics, 9, 465

Wang, Q. D., Nowak, M. A., Markoff, S. B., et al. 2013, Science, 341, 981

Yuan, F., Lin, J., Wu, K., \& Ho, L. C. 2009, MNRAS, 395, 2183

Yuan, F. \& Narayan, R. 2014, ARA\&A A, 52, 529

Yuan, F., Quataert, E., \& Narayan, R. 2003, ApJ, 598, 301

Yuan, Q. \& Wang, Q. D. 2016, MNRAS, 456, 1438

Yusef-Zadeh, F., Roberts, D., Wardle, M., Heinke, C. O., \& Bower, G. C. 2006b, ApJ, 650, 189

Yusef-Zadeh, F., Wardle, M., Dodds-Eden, K., et al. 2012, AJ, 144, 1

Zubovas, K., Nayakshin, S., \& Markoff, S. 2012, MNRAS, 421, 1315 